\section{Free-floating right atrial thrombus with acute pulmonary embolism}

A 70-year-old man had an episode of dyspnoea followed by syncope 2 months after a stroke. Deep venous thrombosis was found in his right lower extremity by venous Doppler ultrasound. Transoesophageal echocardiography revealed a $33 \times 38 \mathrm{~mm}$ highly mobile mass floating in his right atrium with no attachment to any atrium structure (fig 1A). The right heart chambers were dilated with mild tricuspid valve
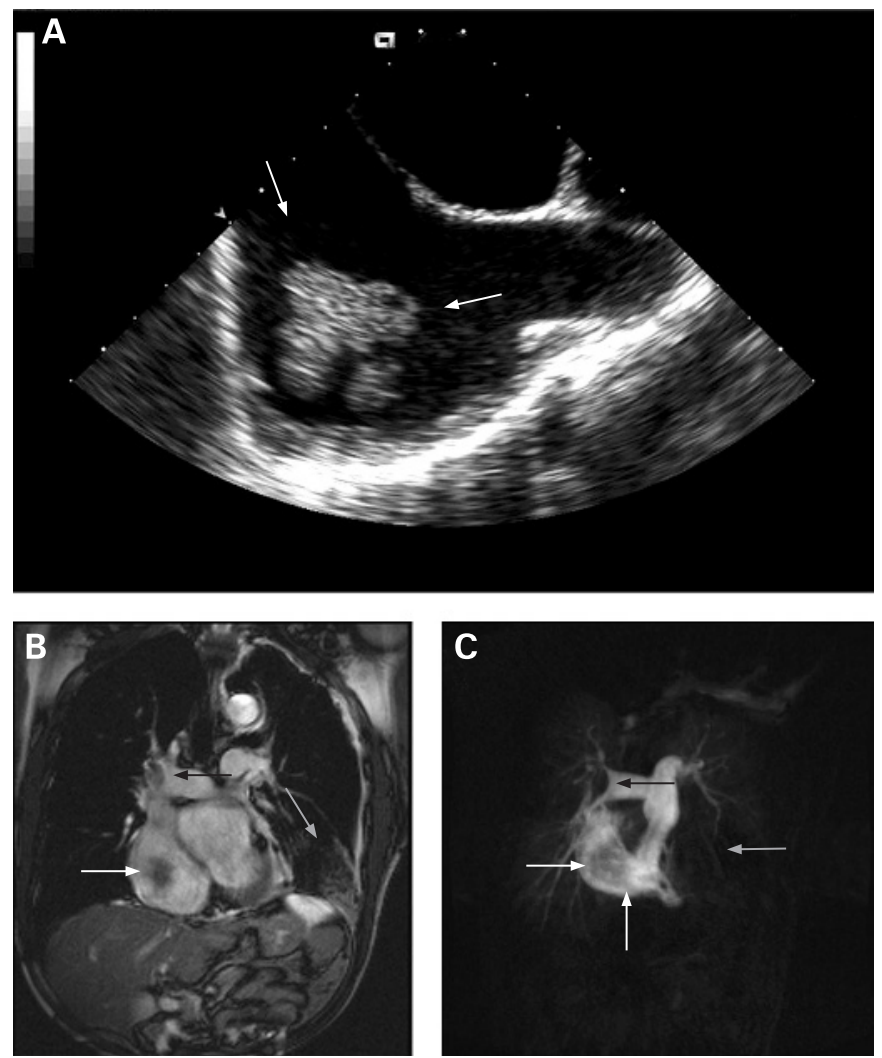

Figure 1 (A) Transoesophageal echocardiogram showing a large thrombus (arrows) floating in the right atrium with no attachment to any heart chamber structure. (B) Cine magnetic resonance imaging (MRI) showing a low-signal floating mass (white arrow) in the right atrium and a large thromboembolus (black arrow) in the right main pulmonary artery. Left lower lobe consolidation can be also noted (grey arrow). (C) Perfusion view of MRI showing the floating right atrial thrombus (white arrows) and right main pulmonary thromboemboli with no perfusion to left lower lobe (grey arrow).

\section{Learning points}

- Nearly $100 \%$ of free-floating right heart thrombi are associated with the presence of pulmonary embolism.

- Cine magnetic resonance imaging is an alternative modality for the diagnosis of right heart free-floating thrombus and pulmonary embolism.

- Surgical thromboembolectomy remains the classic treatment.

regurgitation and a systolic pulmonary artery pressure of $54 \mathrm{~mm} \mathrm{Hg}$. Cine magnetic resonance imaging showed a lowsignal free-floating mass within the right atrium and a large thrombus in the right main pulmonary artery in the same view (fig 1B). No perfusion to the left lower lobe was observed in the perfusion view (fig 1C). Emergency thromboembolectomy was performed. The diagnosis of thrombus was confirmed by histological examination. Two years later an echocardiogram showed a normal heart structure and function.

This case illustrates well the potential life-threatening nature of free-floating right atrial thrombus, and suggests that prompt intervention is merited whenever these lesions are detected. However, the management strategy remains controversial. ${ }^{12}$ Although thrombolysis is advocated first, surgical thromboembolectomy remains the classic treatment, particularly for cases in whom thrombolysis is contraindicated or if thrombolysis is ineffective. Anticoagulation with heparin is more an antithrombotic than a lytic therapy. It is inappropriate as the sole treatment of an impending pulmonary embolism.

\section{Hou, ${ }^{1}$ W Liu, ${ }^{2}$ Z Zhang, ${ }^{3}$ Z Li ${ }^{2}$}

${ }^{1}$ Department of Cardiac Surgery, Beiijng Anzhen Hospital, Capital Medical University, Beijing, China; ${ }^{2}$ Department of Echocardiogram, Beijing Anzhen Hospital, Capital Medical University, Beijing, China; ${ }^{3}$ Department of Radiology, Beijing Anzhen Hospital, Capital Medical University, Beijing, China

Correspondence to: Dr X Hou, Department of Cardiac Surgery, Beijing Anzhen Hospital, Anzhenli, Andingmenwai, Chaoyang District, Beijing, China 100029; houxiaotong@yahoo.com.cn

Competing interests: None.

Patient consent: Obtained.

Received 17 September 2008 Accepted 26 November 2008

Thorax 2009:64:736. doi:10.1136/thx.2008.108217

\section{REFERENCES}

1. Chartier L, Bera J, Delomez $\mathrm{M}$, et al. Free-floating thrombi in the right heart: diagnosis, management, and prognostic indexes in 38 consecutive patients. Circulation 1999:99:2779-83.

2. Rose PS, Punjabi NM, Pearse DB. Treatment of right heart thromboemboli. Chest 2002;121;806-14 